\title{
New efficient synthesis and bioactivity of homoisoflavonoids
}

\author{
Vallabhaneni Madhava Rao, Guri Lakshmi Vasantha Damu, Dega Sudhakar, \\ Vidavaluri Siddaiah, and Chunduri Venkata Rao*
}

Natural Products Division, Department of Chemistry, Sri Venkateswara University, Tirupati-517 502, India

E-mail: cvraosvu@gmail.com

\begin{abstract}
Two naturally occurring homoisoflavonoids (3h,3i) and nine analogs (2a-2i) have been synthesised from appropriately substituted phenols thorough 1-(2-hydroxyphenyl)-3phenylpropane-1-ones (1a-1i). The anti-oxidant and anti-fungal activities were determined by superoxide (NBT) and Agar cup method respectively. The reaction of 1-(2-hydroxyphenyl)-3phenylpropane-1-ones with triethylorthoformate and $70 \%$ perchloric acid followed by aqueous hydrolysis of the intermediate perchlorates, the corresponding homoisoflavones (2a-2i) are obtained in excellent yields. Thereafter, 2a-2i has been converted into homoisoflavanones by subjecting to catalytic hydrogenation. This methodology has been applied for the first time for the synthesis of $( \pm$ )-dihydrobonducellin (3h) and ( \pm )-2'-methoxydihydrobonducellin (3i), which have been isolated from Caesalpinia pulcherrima.
\end{abstract}

Keywords: 2'-Hydroxydihydrochalcones, 3-benzylchromones, dihydrobonducellin, 2'-methoxydihydrobonducellin, Caesalpinia pulcherrima

\section{Introduction}

Homoisoflavonoids were isolated from Eucomis species such as E. bicolour, E. autumnalis, E. punctata, Ophiopogan japanicus, O. jaburan ${ }^{1}$ belongs to Liliaceae family and Caesalpinia sappan, Caesalpinia pulcherrima ${ }^{2}$ belongs to Leguminosae family. These are structurally related to flavonoids and display a wide spectrum of biological activities. 3Benzylidenechromanones known to act as a growth inhibitors of the sporogeneses and the enzymes involved in the infection mechanism of Phytophthora parasitica ${ }^{3}$. Some of the 3benzylidenechromanones display anti-inflammatory, antifungal, antioxidant, anti-aggregating, analgesic, platet, and hypocholestrolenic activities ${ }^{4-8}$. Some natural derivatives display antimutagenic $^{9}$, phosphidiesterase isoenzyme-inhibiting $^{10}$ and antiviral properties ${ }^{11,12}$. On the other hand, various 3-benzyl- chromones posses angioprotective, antiallergic, antihistaminic, 
properties $^{13}$. Their properties turn homoisoflavonoids very interesting targets to organic chemists.

An extensive survey of the literature shows that a few methods of synthesis have been reported for homoisoflavones. (i) 3-Benzylchromones were prepared from 3-benzylidenechromanones by isomerisation of the double bond using Pd-C at $250{ }^{\circ} \mathrm{C}^{14}$, potassium tert-butarate ${ }^{1 \mathrm{~d}}$, rhodium trichloride ${ }^{15}$, or Raney nickel ${ }^{16}$. These reactions lead generally to low yields. (ii) Hydrogenation of chalcones followed by one carbon extension using ethyl formate/sodium ${ }^{17}$ or methanesulfonyl chloride/DMF ${ }^{18}$.

3-Benzylchromanones are usually synthesized by the catalytic reduction of 3-benzylidinechromanones. But their preparation is limited by the availability of the starting 3benzylchromones. These were also synthesized from $o$-hydroxydihydro- chalcones in low yields by one carbon extension using ethoxymethylchloride ${ }^{19}$ paraformaldehyde and aq.diethylamine ${ }^{20}$ or 3-benzyl-4-hydroxycoumarins by the hydroboration followed by chromic acid oxidation ${ }^{21}$.

In our research program, we are interested in synthesis and bioactivity evaluation of homoisoflavonoids ${ }^{5,6}$ and an increasing demand for a short and efficient method prompted us to develop a simple and general method for the synthesis homoisoflavones.

\section{Results and Discussions}

During the course of our investigations of new synthetic routes to homoisoflavonoids, we have found that a novel and efficient approach to homoisoflavones (Scheme 1). The method involves homoisoflavones which are prepared by the treatment of 2'-hydroxydihydrochalcones with triethylorthoformate and $70 \%$ perchloric acid followed by aqueous hydrolysis of the intermediate perchlorates $^{22}$.

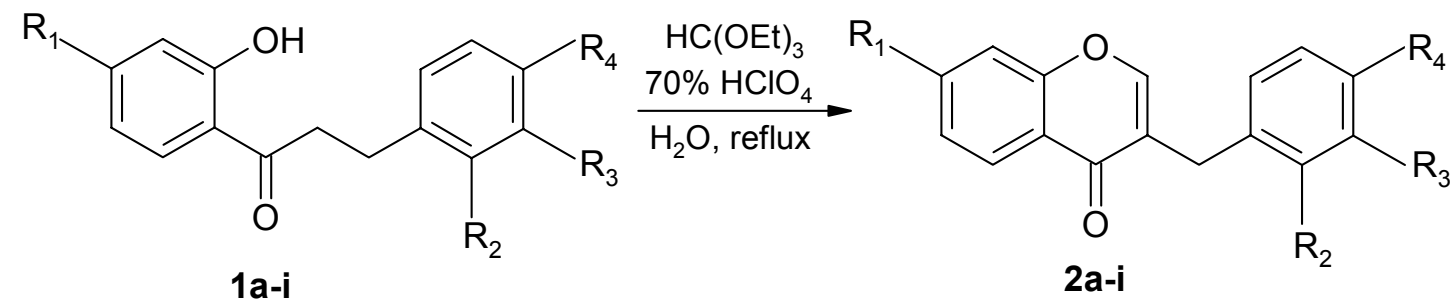

For compounds 1a-i \& 2a-i
a) $\mathrm{R}_{1}=\mathrm{R}_{2}=\mathrm{R}_{3}=\mathrm{R}_{4}=\mathrm{H}$
f) $\mathrm{R}_{1}=\mathrm{OBz}, \mathrm{R}_{2}=\mathrm{R}_{3}=\mathrm{H}, \mathrm{R}_{4}=\mathrm{OCH}_{3}$
b) $\mathrm{R}_{1}=\mathrm{R}_{2}=\mathrm{R}_{3}=\mathrm{H}, \mathrm{R}_{4}=\mathrm{OCH}_{3}$
g) $\mathrm{R}_{1}=\mathrm{R}_{3}=\mathrm{R}_{4}=\mathrm{OCH}_{3}, \mathrm{R} 2=\mathrm{H}$
c) $\mathrm{R}_{1}=\mathrm{R}_{2}=\mathrm{H}, \mathrm{R}_{3}=\mathrm{R}_{4}=\mathrm{OCH}_{3}$
h) $\mathrm{R}_{1}=\mathrm{OH}, \mathrm{R}_{2}=\mathrm{R}_{3}=\mathrm{H}, \mathrm{R}_{4}=\mathrm{OCH}_{3}$
d) $\mathrm{R}_{1}=\mathrm{R}_{2}=\mathrm{R}_{3}=\mathrm{H}, \mathrm{R}_{4}=\mathrm{Cl}$
i) $\mathrm{R}_{1}=\mathrm{OH}, \mathrm{R}_{3}=\mathrm{H}, \mathrm{R}_{2}=\mathrm{R}_{4}=\mathrm{OCH}_{3}$
e) $\mathrm{R}_{1}=\mathrm{OCH}_{3}, \mathrm{R}_{2}=\mathrm{R}_{3}=\mathrm{R}_{4}=\mathrm{H}$

\section{Scheme 1}


However, in our experiments when the reaction was performed with the methoxy, chloro substituent derivatives homoisoflavones were obtained in excellent yields in contrast to the case of hydroxyl derivatives. All the structures of the products were established by comparison of analytical data (IR, NMR and MS) of those reported authentic samples.

The synthesis of 3-benzylchromanones was achieved by catalytic hydrogenation of corresponding 3-benzylchromones (Scheme 2). Dihydrobonducellin (3h) and 2'-Methoxydihydrobonducellin (3i), isolated recently from Caesalpinia pulcherrima ${ }^{23}$ were synthesized by following the above methodology in $78 \%, 75 \%$ Yields respectively. The spectral data of synthetic $\mathbf{3 h}, \mathbf{3 i}$ were identical with those of natural products. The over all yields of homoisoflavanones are higher than those obtained with previously reported procedures.<smiles>[R]C1=CC=C2C(=O)C(Cc3ccc([R20])c([R])c3[R2])=CC=C2OC=C1CC1COc2cc([R])ccc2C1=O</smiles>

3h: $\mathrm{R}_{1}=\mathrm{OH}, \mathrm{R}_{2}=\mathrm{R}_{3}=\mathrm{H}, \mathrm{R}_{4}=\mathrm{OCH}_{3}$

3i: $\mathrm{R}_{1}=\mathrm{OH}, \mathrm{R}_{3}=\mathrm{H}, \mathrm{R}_{2}=\mathrm{R}_{4}=\mathrm{OCH}_{3}$

\section{Scheme 2}

\section{Antioxidant activity}

The 3-benzylchromones exhibited better antioxidant activity than the known antioxidants, namely, vitamin $\mathrm{C}\left(\mathrm{IC}_{50}=852 \mu \mathrm{M}\right)$, vitamin $\mathrm{E}\left(\mathrm{IC}_{50}=726 \mu \mathrm{M}\right), \mathrm{BHA}\left(\mathrm{IC}_{50}=966 \mu \mathrm{M}\right)$ and $\mathrm{BHT}$ $\left(\mathrm{IC}_{50}=381 \mu \mathrm{M}\right)($ Table 1$)$. The antioxidant activity studies revealed that the 3-benzylchromones with phenolic hydroxyl group (2h\&2i) exhibited good activity than the compounds with methoxyl groups (2e\&2g). The presence of double bond between $\mathrm{C}_{2}-\mathrm{C}_{3}$ has major influence on the activity of compounds. For example, 3-benzylchromones having saturation between $\mathrm{C}_{2}-\mathrm{C}_{3}$ (3h\&3i) exhibited very poor antioxidant activity than the compounds with $\mathrm{C}_{2}-\mathrm{C}_{3}$ double bond $(\mathbf{2 h} \& \mathbf{2 i})$. These results clearly indicated that the compounds, in order to exhibit good antioxidant activity must contain $\mathrm{C}_{2}-\mathrm{C}_{3}$ double bond and hydroxyl groups. The results of inhibition concentration $\left(\mathrm{IC}_{50}\right.$ in $\mu \mathrm{M}$ ) of the 3-benzylchromones have been incorporated in Table 1.

\section{Antifungal activity}

Among the eleven 3-benzylchromone derivatives examined most showed good antifungal activity against Aspergillus niger and Penicillum chrysogenium. The compounds $\mathbf{2 d}, \mathbf{2 f}, \mathbf{2 g}, \mathbf{2 h}$ and $\mathbf{2} \mathbf{i}$ showed good activity against two strains (zone of inhibition $\geq 5 \mathrm{~mm}$ ) and indicated that the presence of 7-oxygenation (i.e. methoxy or hydroxyl group at $7^{\text {th }}$ position) and methoxy or chloro substituents at C-4' were required for effective antifungal activity. The compound $\mathbf{2 i}$ showed highest activity among all due to the presence of 2'-oxygenation along with 7, 4'- 
oxygenation. The two 3-benzylchromones (3h \& 3i) with saturation at $\mathrm{C}_{2}-\mathrm{C}_{3}$ position exhibited less activity than corresponding benzylchromones. From these results it was clear that the compounds with chloro, methoxy and hydroxyl substituents at suitable positions and presence of double at $\mathrm{C}_{2}-\mathrm{C}_{3}$ position exhibited effective antifungal activity. The results have been showed in Table 2.

Table 1. Antioxidant activity 3-benzylchromones

\begin{tabular}{cccc}
\hline Compd. & $\mathrm{R}_{1}$ & $\mathrm{R}_{2}$ & $\mathrm{IC}_{50} \mu \mathrm{M}$ \\
\hline $\mathbf{2 a}$ & - & - & $\mathrm{ND}$ \\
$\mathbf{2 b}$ & - & $4^{\prime}-\mathrm{OCH}_{3}$ & 240 \\
$\mathbf{2 c}$ & - & $3^{\prime}, 4^{\prime}-\mathrm{Di}-\mathrm{OMe}$ & 238 \\
$\mathbf{2 d}$ & - & $4^{\prime}-\mathrm{Cl}$ & 251 \\
$\mathbf{2 e}$ & $7-\mathrm{OMe}$ & - & 243 \\
$\mathbf{2 f}$ & $7-\mathrm{OBz}$ & $4 '-\mathrm{OMe}$ & 220 \\
$\mathbf{2 g}$ & $7-\mathrm{OMe}$ & 3', $^{\prime}-\mathrm{Di}-\mathrm{OMe}$ & 232 \\
$\mathbf{2 h}$ & $7-\mathrm{OH}$ & $4^{\prime}-\mathrm{OMe}$ & 162 \\
$\mathbf{2 i}$ & $7-\mathrm{OH}$ & $2^{\prime}, 4^{\prime}-\mathrm{Di}-\mathrm{OMe}$ & 158 \\
$\mathbf{3 h}$ & $7-\mathrm{OH}$ & $4^{\prime}-\mathrm{OMe}$ & 342 \\
$\mathbf{3 i}$ & $7-\mathrm{OH}$ & $2^{\prime}, 4^{\prime}-\mathrm{Di}-\mathrm{OMe}$ & 325 \\
Vitamin C & & & 852 \\
Vitamin E & & & 726 \\
BHA & & & 966 \\
BHT & & & 381 \\
\hline
\end{tabular}

ND- Not Determined.

Table 2. Antifungal activity of 3-benzylchromones

\begin{tabular}{|c|c|c|c|c|c|}
\hline \multirow[b]{2}{*}{ Compound } & \multirow[b]{2}{*}{$\mathrm{R}_{1}$} & \multirow[b]{2}{*}{$\mathrm{R}_{2}$} & \multirow[b]{2}{*}{$\begin{array}{l}\text { Conc. } \\
(\mu \mathrm{L})\end{array}$} & \multicolumn{2}{|c|}{ Zone of inhibition (mm) } \\
\hline & & & & $\begin{array}{l}\text { Aspergillus } \\
\text { niger }\end{array}$ & $\begin{array}{l}\text { Penicillum } \\
\text { chrysogenium }\end{array}$ \\
\hline \multirow{2}{*}{$2 \mathbf{a}$} & \multirow[b]{2}{*}{ - } & \multirow{2}{*}{ - } & 10 & \multirow{2}{*}{ ND } & \multirow{2}{*}{ ND } \\
\hline & & & 20 & & \\
\hline \multirow{2}{*}{$2 b$} & \multirow[b]{2}{*}{-} & \multirow{2}{*}{$4^{\prime}-\mathrm{OCH}_{3}$} & 10 & 2 & 2 \\
\hline & & & 20 & 2 & 2 \\
\hline \multirow{2}{*}{$2 c$} & \multirow{2}{*}{-} & \multirow{2}{*}{ 3',4'-Di-OMe } & 10 & 2 & 3 \\
\hline & & & 20 & 3 & 3 \\
\hline \multirow{2}{*}{ 2d } & \multirow{2}{*}{ - } & \multirow{2}{*}{$4^{\prime}-\mathrm{Cl}$} & 10 & 4 & 4 \\
\hline & & & 20 & 7 & 8 \\
\hline \multirow{2}{*}{$2 e$} & \multirow{2}{*}{ 7-OMe } & \multirow{2}{*}{-} & 10 & 2 & 3 \\
\hline & & & 20 & 4 & 3 \\
\hline
\end{tabular}




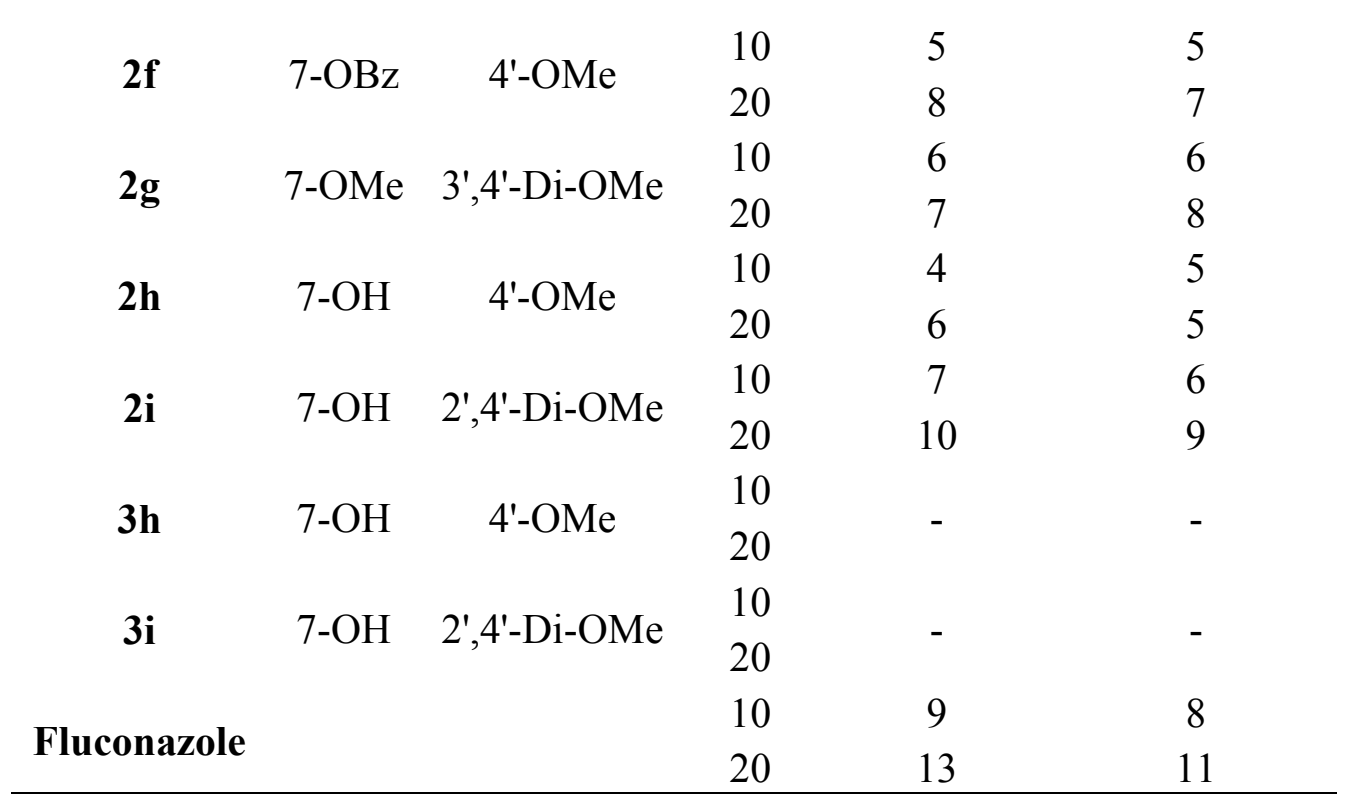

ND- Not Determined. - No Activity.

\section{Conclusions}

In conclusion, we have described an efficient method for the synthesis of homoisoflavones by one carbon extension using triethylorthoformate and 70\% perchloric acid followed by aqueous hydrolysis of the intermediate perchlorates in excellent yields. They were found to be active in vitro against the pathogenic fungi Aspergilus niger and Penicillium chrysogenium and also they exhibit some antioxidant activity. We have also accomplished first time the synthesis of $( \pm)$ dihydrobonducellin and $( \pm)-2^{\prime}-$ methoxy-dihydrobonducellin from Caesalpinia pulcherrima.

\section{Experimental Section}

General Procedures. Melting points were recorded on a Mel-Temp melting point apparatus, in open capillaries and are uncorrected. IR spectra were recorded on a Perkin-Elmer BX1 FTIR Spectrophotometer and ${ }^{1} \mathrm{H}$ NMR $(400 \mathrm{MHz})$, spectra were recorded on a Bruker AMX $400 \mathrm{MHz}$ NMR spectrometer using TMS as internal standard and the values for chemical shifts $(\delta)$ being given in ppm and coupling constants $(J)$ in Hertz $(\mathrm{Hz})$. Mass spectra were recorded on an Agilent 1100 LC/MSD. Acme silica gel G and silica gel (100-200 mesh) were used for analytical TLC and column chromatography, respectively. 


\section{Synthesis of homoisoflavones(2a-i). General procedure}

2'-Hydroxydihydrochalcones were prepared by following the general procedure ${ }^{5}$ from commercially available phenols and 3-phenylpropionic acids using ethereal solution of borontrifluoride. A suspension of 2'-hydroxydihydrochalcone $(0.5 \mathrm{~g}, 2.21 \mathrm{mmol})$ in triethylorthoformate $(2.9 \mathrm{~mL})$ was placed in $10 \mathrm{ml}$ round bottom flask. While the suspension was stirred with a magnetic stirrer, $70 \%$ perchloric acid $(0.33 \mathrm{~mL})$ was added from an addition funnel over a 3min period. Thick dark solution that resulted was slightly warm to the touch, and it was stirred until it cooled to room temperature (about $40 \mathrm{~min})$. Anhydrous ethyl ether $(10 \mathrm{~mL})$ was added and brown precipitate formed. This solid was filtered, dissolved in hot water $(10 \mathrm{~mL})$, and heated at reflux for $5 \mathrm{~min}$. The mixture was then allowed to cool to room temperature then the solution was extracted with EtOAc (3 X $50 \mathrm{~mL}$ ) and the combined EtOAc layer was washed with water $(20 \mathrm{~mL})$, brine $(20 \mathrm{~mL})$ and dried over sodium sulphate. The residue obtained after evaporation of the solvent was chromatographed over silica gel column using hexane-EtOAc mixtures as eluent to give 2a-i.

3-Benzylchromone (2a). Mp: $108-109{ }^{\circ} \mathrm{C}$ (lit. ${ }^{13}$ Mp. $110{ }^{\circ} \mathrm{C}$ ); Yield $83 \%$ (433 mg); Anal.Calcd. for $\mathrm{C}_{16} \mathrm{H}_{12} \mathrm{O}_{2}$ : C, 81.35; H, 5.08\%, Found: C, 81.30; H, 5.12\%. IR (KBr) $v_{\max }$ :1637, 1610, 1238, 1072, $980 \mathrm{~cm}^{-1}$; ${ }^{1} \mathrm{H}-\mathrm{NMR}$ (DMSO- $\left.d_{6}\right): \delta 8.10(1 \mathrm{H}, \mathrm{s}, \mathrm{H}-2), 7.68(1 \mathrm{H}, \mathrm{dd}$, $\mathrm{J}=8.8,2.7 \mathrm{~Hz}, \mathrm{H}-5), 7.36(5 \mathrm{H}, \mathrm{m}, \mathrm{Ar}-\mathrm{H}), 7.0-6.85(2 \mathrm{H}, \mathrm{m}, \mathrm{H}-6,7), 6.78(1 \mathrm{H}, \mathrm{dd}, \mathrm{J}=8.5,2.3 \mathrm{~Hz}, \mathrm{H}-$ 8), 3.85(2H, s, H-9); ${ }^{13} \mathrm{C}-\mathrm{NMR}$ (DMSO- $\left.d_{6}\right): \delta 176.3(\mathrm{C}-4), 156.2(\mathrm{C}-8 \mathrm{a}), 152.8(\mathrm{C}-2), 138.6\left(\mathrm{C}-1^{\prime}\right)$, 133.2(C-7), 129.2(C-2',6'), 128.6(C-3',5'), 126.3(C-4'), 125.6(C-6), 124.6(C-5), 124.4(C-3), 123.6 (C-4a), 117.7(C-8), 30.8(C-9). LC-MS (ESI, negative ion mode): $\mathrm{m} / z 235$ (M-H) ${ }^{-}$

3-(4-Methoxybenzyl)chromone (2b). Mp: 101-102 ${ }^{\circ} \mathrm{C}$ (lit. ${ }^{13} \mathrm{Mp} .101{ }^{\circ} \mathrm{C}$ ); Yield 81\% (420 mg); Anal.Calcd. for $\mathrm{C}_{17} \mathrm{H}_{14} \mathrm{O}_{3}: \mathrm{C}, 76.9 ; \mathrm{H}, 5.26 \%$. Found: C, 76.65; H, 5.30\%. IR (KBr) v $v_{\max }$ : 3017, 2925, 1642, 1607, 1240, 1172, $1068 \mathrm{~cm}^{-1}$; ${ }^{1} \mathrm{H}-\mathrm{NMR}$ (DMSO- $\left.d_{6}\right): \delta 8.06(1 \mathrm{H}, \mathrm{s}, \mathrm{H}-2), 7.82(1 \mathrm{H}$, dd, J-8.8, $2.7 \mathrm{~Hz}, \mathrm{H}-5), 7.33\left(2 \mathrm{H}, \mathrm{d}, \mathrm{J}=9.5 \mathrm{~Hz}, \mathrm{H}-2^{\prime}, 6^{\prime}\right), 7.10\left(2 \mathrm{H}, \mathrm{d}, \mathrm{J}=9.5 \mathrm{~Hz}, \mathrm{H}-3^{\prime}, 5^{\prime}\right), 7.1-$ 6.90(2H, m, H-6, 7), 6.80(1H, dd, J=8.5, $2.3 \mathrm{~Hz}, \mathrm{H}-8), 3.82(3 \mathrm{H}, \mathrm{s}, \mathrm{Ar}-\mathrm{OMe}), 3.76(2 \mathrm{H}, \mathrm{s}, \mathrm{H}-9)$; ${ }^{13} \mathrm{C}-\mathrm{NMR}\left(\mathrm{DMSO}-d_{6}\right): \delta 176.2(\mathrm{C}-4), 158\left(\mathrm{C}-4^{\prime}\right), 156.2(\mathrm{C}-8 \mathrm{a}), 152.8(\mathrm{C}-2), 133.2(\mathrm{C}-7), 130.3(\mathrm{C}-$ 1'), 129.7(C-2',6'), 125.8(C-6), 124.7(C-3), 123.6(C-4a), 117.7(C-8), 113.7(C-3',5'), 54.9(OMe4'), 30.7(C-9); LC-MS (ESI, negative ion mode): $m / z 265(\mathrm{M}-\mathrm{H})^{-}$.

3-(3, 4-Dimethoxybenzyl)chromone (2c). Mp:114-116 ${ }^{\circ} \mathrm{C}$.(lit. $\left.{ }^{13} \mathrm{Mp} .115{ }^{\circ} \mathrm{C}\right)$;Yield $85 \%(439$ mg). Anal.Calcd. for $\mathrm{C}_{18} \mathrm{H}_{16} \mathrm{O}_{4}$ : C, 72.97; $\mathrm{H}, 5.40 \%$. Found: C, 73.00; H, 5.52\%.

$\operatorname{IR}(\mathrm{KBr}) v_{\max }: 2937,2876,1632 ， 1260,1172,1138,1027,965 \mathrm{~cm}^{-1} ;{ }^{1} \mathrm{H}-\mathrm{NMR}\left(\mathrm{DMSO}-d_{6}\right)$ : $\delta$ 8.01(1H, s, H-2), 7.87(1H, dd, J=8.8, $2.7 \mathrm{~Hz}, \mathrm{H}-5), 7.20-6.78(6 \mathrm{H}, \mathrm{m}, \mathrm{Ar}-\mathrm{H}), 3.89$ (3H, s, Ar$\mathrm{OMe}$ ), 3.85(3H, s, Ar-OMe), 3.78(2H, s, H-9); ${ }^{13} \mathrm{C}-\mathrm{NMR}$ (DMSO- $\left.d_{6}\right): \delta 176.5(\mathrm{C}-4), 156.2(\mathrm{C}-$ 8a), 152.6(C-2), 148.9(C-4'), 147.8(C-3'), 133.0(C-7), 130.2(C-1'), 125.6(C-6), 124.5(C-3,5), 123.7(C-4a), 120.1(C-6'), 177.8(C-8), 122.2(C-5'), 111.2(C-2'), 55.5(OMe-4'), 55.3(OMe-3'), 30.9(C-9); LC-MS (ESI, negative ion mode): $\mathrm{m} / 2295(\mathrm{M}-\mathrm{H})^{-}$.

3-(4-Chlorobenzyl)chromone (2d). Mp:139-140 ${ }^{\circ} \mathrm{C}$. (lit. ${ }^{13} \mathrm{Mp} .140{ }^{\circ} \mathrm{C}$ ); Yield 80 \% (415 mg); Anal.Calcd. for $\mathrm{C}_{16} \mathrm{H}_{11} \mathrm{O}_{2} \mathrm{Cl}$ : C, 70.97; H, 4.06; Cl, 13.12\%. Found: $\mathrm{C}, 70.77 ; \mathrm{H}, 4.10 ; \mathrm{Cl}$, 
13.09\%. IR (KBr) v $v_{\max }: 1635,1239,1143,1068,768 \mathrm{~cm}^{-1} ;{ }^{1} \mathrm{H}-\mathrm{NMR}\left(\mathrm{DMSO}-d_{6}\right): \delta 8.20(1 \mathrm{H}, \mathrm{dd}$, $\mathrm{J}=8.8,2.7 \mathrm{~Hz}, \mathrm{H}-5), 7.61(1 \mathrm{H}, \mathrm{s}, \mathrm{H}-2), 7.34\left(2 \mathrm{H}, \mathrm{d}, \mathrm{J}=9.5 \mathrm{~Hz}, \mathrm{H}-2^{\prime}, 6^{\prime}\right), 7.15(2 \mathrm{H}, \mathrm{d}, \mathrm{J}=9.5 \mathrm{~Hz}, \mathrm{H}-3$ ', $\left.5^{\prime}\right), 7.0-6.87(2 \mathrm{H}, \mathrm{m}, \mathrm{H}-6,7), 6.85(1 \mathrm{H}, \mathrm{dd}, \mathrm{J}=8.6,2.4 \mathrm{~Hz}, \mathrm{H}-8), 3.76(2 \mathrm{H}, \mathrm{s}, \mathrm{H}-9)$; ${ }^{13} \mathrm{C}-\mathrm{NMR}$ $\left(\mathrm{DMSO}-d_{6}\right): \delta 176.3(\mathrm{C}-4), 156.1(\mathrm{C}-8 \mathrm{a}), 152.5(\mathrm{C}-2), 136.2\left(\mathrm{C}-1^{\prime}\right), 133.2(\mathrm{C}-7), 131.7\left(\mathrm{C}-4^{\prime}\right)$, 130.7(C-5), 129.1(C-2',6'), 125.7(C-3',5'), 124.8(C-4a), 123.6(C-6), 117.7 (C-8), 115.7(C-3), 30.9(C-9); LC-MS (ESI, negative ion mode): $m / z 269.5(\mathrm{M}-\mathrm{H})^{-}$.

7-Methoxy-3-benzylchromone (2e). Mp: $127-129{ }^{\circ} \mathrm{C}$. (lit. ${ }^{13} \mathrm{Mp} .131{ }^{\circ} \mathrm{C}$ ); Yield $85 \%$ (441 mg). Anal.Calcd. for $\mathrm{C}_{17} \mathrm{H}_{14} \mathrm{O}_{3}$ : C, 76.69; H, 5.26\%. Found: C, 76.63; H, 5.31\%. IR (KBr) v $v_{\max }$ : 3024, 2917, 1640, 1609, 1268, 1183, 1161, 1070, $1025 \mathrm{~cm}^{-1} ;{ }^{1} \mathrm{H}-\mathrm{NMR}\left(\mathrm{CDCl}_{3}\right): \delta 8.13(1 \mathrm{H}, \mathrm{d}, \mathrm{J}=8.9$ Hz, H-5), 7.51 (1H, s, H-2), 7.22-723 (5H, m, Ar-H), 6.95 (1H, dd, J=8.9, 2.2 Hz, H-6), 6.77 $(1 \mathrm{H}, \mathrm{d}, \mathrm{J}=2.2 \mathrm{~Hz}, \mathrm{H}-8), 3.88(3 \mathrm{H}, \mathrm{s}, \mathrm{Ar}-\mathrm{OMe}) ; 3.80(2 \mathrm{H}, \mathrm{s}, \mathrm{H}-9) ;{ }^{13} \mathrm{C}-\mathrm{NMR}\left(\mathrm{CDCl}_{3}\right)$ : $\delta 176.2(\mathrm{C}-$ 4), 163.4(C-7), 158.1(C-8a), 151.9(C-2), 138.6 (C-1'), 128.2(C-2',3',5',6'), 127.0(C-5), 126.3(C4'), 124.5(C-3), 117.6(C-4a), 114.5(C-6), 100.2(C-8), 55.3(OMe-7), 30.8(C-9); LC-MS (ESI, positive ion mode): $m / z 267(\mathrm{M}+\mathrm{H})^{+}$.

7-Benzyloxy-3-(4-methoxybenzyl)chromone (2f). Mp:172-173 ${ }^{\circ} \mathrm{C}$ (lit. ${ }^{24}$ M.P. $173{ }^{\circ} \mathrm{C}$ ); Yield $84 \%$ (431 mg). Anal.Calcd. for $\mathrm{C}_{24} \mathrm{H}_{20} \mathrm{O}_{4}$ : C, 77.4; H, $5.4 \%$. Found: C, 77.1; H, $5.6 \%$. IR $(\mathrm{KBr}) v_{\max }: 2920,1652,1604,1248,1160,1129,1094,1032 \mathrm{~cm}^{-1} ;{ }^{1} \mathrm{H}-\mathrm{NMR}$ (DMSO- $\left.d_{6}\right): \delta 8.05$ $(1 \mathrm{H}, \mathrm{d}, \mathrm{J}=9.5 \mathrm{~Hz}, \mathrm{H}-5), 7.41(1 \mathrm{H}, \mathrm{s}, \mathrm{H}-2), 7.30-7.34$ (5H, s, Ar-H), 7.32 (2H, d, J=9.5 Hz, H2',6'), 7.09 (2H, d, J=9.5 Hz, H-3',5'), 6.92 (1H, dd, J=2.3 \& 9.5 Hz, H-6), 6.82 (1H, s, H-8), $5.09\left(2 \mathrm{H}, \mathrm{s},-\mathrm{OCH}_{2}-\mathrm{Ar}\right), 3.72(3 \mathrm{H}, \mathrm{s}, \mathrm{Ar}-\mathrm{OMe}), 3.69(2 \mathrm{H}, \mathrm{s}, \mathrm{H}-9) ;{ }^{13} \mathrm{C}-\mathrm{NMR}$ (DMSO- $\left.d_{6}\right): \delta$ 176.3(C-4), 164.4(C-7), 157.8(C-8a), 157.4(C-4'), 152.1(C-2), 140.5(C-1"), 130.2(C-5), 129.4(C$\left.2^{\prime}, 6^{\prime}\right), 126.6\left(\mathrm{C}-1^{\prime}\right), 125.8$ (C-3",5"), 124.5(C-4"), 124.0(C-2",6"), 123.2(C-4a), 116.3(C-3), 114.6(C-3',5'), 111.8 (C-6), 100.4(C-8), 63.9 (-O-CH2-), 54.9(OMe-4'), 30.4(C-9);

LC-MS (ESI, negative ion mode): $m / z 371(\mathrm{M}-\mathrm{H})^{-}$.

7-Methoxy-3-(3, 4-dimethoxybenzyl)chromone (2g). Mp: $128-130{ }^{\circ} \mathrm{C}$; Yield $88 \%$ (453 mg). Anal.Calcd. for $\mathrm{C}_{19} \mathrm{H}_{18} \mathrm{O}_{5}$ : C, 69.93; H, 5.52\%.Found: C, 69.88; H, 5.52\%. IR (KBr) v $v_{\max }$ : 3074, 2957, 1637, 1608, 1275, 1172, 1135, 1096, $1024 \mathrm{~cm}^{-1} ;{ }^{1} \mathrm{H}-\mathrm{NMR}\left(\mathrm{CDCl}_{3}\right): \delta 8.13(1 \mathrm{H}, \mathrm{d}, \mathrm{J}=8.8$ $\mathrm{Hz}, \mathrm{H}-5), 7.51(1 \mathrm{H}, \mathrm{s}, \mathrm{H}-2), 6.95(1 \mathrm{H}, \mathrm{dd}, \mathrm{J}=8.8,2.2 \mathrm{~Hz}, \mathrm{H}-6), 6.81-6.83(3 \mathrm{H}, \mathrm{m}, \mathrm{Ar}-\mathrm{H}), 6.78(1 \mathrm{H}$, $\mathrm{d}, \mathrm{J}=2.2 \mathrm{~Hz}, \mathrm{H}-8), 3.88(3 \mathrm{H}, \mathrm{s}, \mathrm{Ar}-\mathrm{OMe}), 3.86(3 \mathrm{H}, \mathrm{s}, \mathrm{Ar}-\mathrm{OMe}), 3.86(3 \mathrm{H}, \mathrm{s}, \mathrm{Ar}-\mathrm{OMe}), 3.74(2 \mathrm{H}, \mathrm{s}$, $\mathrm{H}-9) ;{ }^{13} \mathrm{C}-\mathrm{NMR}\left(\mathrm{CDCl}_{3}\right): \delta$ 176.0(C-4), 162.2(C-7), 157.4(C-8a), 152.3(C-2), 148.5(C-3'), 147.2(C-4'), 131.6(C-5), 127.6(C-1'), 123.4(C-6'), 120.5(C-4a), 116.3(C-3), 114.7(C-5'), 112.2(C-2'), 111.8(C-6), 102.3(C-8), 55.4(OMe-7), 55.3(OMe-4'), 55.1(OMe-3'), 30.5(C-9); LCMS (ESI, positive ion mode): $m / z 327(\mathrm{M}+\mathrm{H})^{+}$.

7-Hydroxy-3-(4-methoxybenzyl)chromone (2h). Mp: $162-164^{\circ} \mathrm{C}$;Yield $88 \%$ (456 mg); Anal.Calcd. for $\mathrm{C}_{17} \mathrm{H}_{14} \mathrm{O}_{4}$ : C, 72.33; H, 5.00\%. Found: C, 72.29; H, 5.04\%. IR (KBr) v $v_{\max }$ : 3433, $1631,1248,1161,1132,1096,1034 \mathrm{~cm}^{-1}$; ${ }^{1} \mathrm{H}-\mathrm{NMR}$ (DMSO- $\left.d_{6}\right): \delta 10.55(1 \mathrm{H}, \mathrm{s}, \mathrm{Ar}-\mathrm{OH})$, 7.96(1H, s, H-2), 7.87(1H, d, J=8.8 Hz, H-5), 7.19(2H, d, J=8.3 Hz, H-2',6'), 6.86(1H, dd, J=8.8, $2.0 \mathrm{~Hz}, \mathrm{H}-6), 6.80$ (2H, d, J=8.3 Hz, H-3',5'),6.71 (1H, d, J=2.0 Hz, H-8), 3.72 (3H, s, Ar-OMe), $3.61(2 \mathrm{H}, \mathrm{s}, \mathrm{H}-9) ;{ }^{13} \mathrm{C}-\mathrm{NMR}\left(\mathrm{DMSO}-d_{6}\right): \delta 176.2(\mathrm{C}-4), 162.2(\mathrm{C}-7), 157.8(\mathrm{C}-8 \mathrm{a}), 157.6\left(\mathrm{C}-4^{\prime}\right)$, 152.1(C-2), 130.6(C-5), 129.4(C-2',6'), 126.6(C-1'), 123.5(C-4a), 116.3(C-3), 114.6(C-3',5'), 
113.4 (C-6), 102.0(C-8), 54.7(OMe-4'), 30.2(C-9); LC-MS (ESI, negative ion mode): $m / z 281$ $(\mathrm{M}-\mathrm{H})^{-}$.

7-Hydroxy-3-(2,4-dimethoxybenzyl)chromone (2i). Mp: $183{ }^{\circ} \mathrm{C}$; Yield 78\% (403 mg); Anal.Calcd. for $\mathrm{C}_{18} \mathrm{H}_{16} \mathrm{O}_{5}$ : C, 69.22; H, 5.16\%. Found: C, 69.18; H, 5.20\%. IR (KBr) v $\max$ : 3224, $1631,1241,1207,1158,1124,1040 \mathrm{~cm}^{-1}$; ${ }^{1} \mathrm{H}-\mathrm{NMR}\left(\mathrm{DMSO}-d_{6}\right): \delta 10.72(1 \mathrm{H}, \mathrm{s}, \mathrm{Ar}-\mathrm{OH})$, 7.86(1H, d, J=8.8 Hz, H-5), 7.85(1H, s, H-2), 7.02(1H, d, J=8.3 Hz, H-6'), 6.88(1H, dd, J=8.8, $2.0 \mathrm{~Hz}, \mathrm{H}-6), 6.79(1 \mathrm{H}, \mathrm{d}, \mathrm{J}=2.0 \mathrm{~Hz}, \mathrm{H}-8), 6.51\left(1 \mathrm{H}, \mathrm{d}, \mathrm{J}=2.1 \mathrm{~Hz}, \mathrm{H}-3^{\prime}\right), 6.42(1 \mathrm{H}, \mathrm{dd}, \mathrm{J}=8.3,2.1$ $\left.\mathrm{Hz}, \mathrm{H}-5^{\prime}\right), 3.77(3 \mathrm{H}, \mathrm{s}, \mathrm{Ar}-\mathrm{OMe}), 3.70(3 \mathrm{H}, \mathrm{s}, \mathrm{Ar}-\mathrm{OMe}), 3.51(2 \mathrm{H}, \mathrm{s}, \mathrm{H}-9)$; ${ }^{13} \mathrm{C}-\mathrm{NMR}$ (DMSO- $\left.d_{6}\right)$ : $\delta$ 175.6(C-4), 161.9(C-7), 159.0(C-2'), 157.6 (C-4',8a), $152.0 \quad$ (C-2), 130.8(C-5), 130.4(C-6'), 123.4(C-1'), 117.5(C-4a), 116.6(C-3), 113.4(C-6), 106.9(C-5'), 102.0(C-8), 100.7(C-3'), 55.0(OMe-2'), 54.7(OMe-4'), 25.3 (C-9); LC-MS (ESI, negative ion mode): $m / z 311(\mathrm{M}-\mathrm{H})^{-}$.

\section{Synthesis of homoisoflavanones (3h \& 3i). General Procedure}

A solution of homoisoflavones $(\mathbf{2 h}, \mathbf{2 i})(500 \mathrm{mg})$ in ethyl acetate $(40 \mathrm{~mL})$ was treated with $10 \%$ Pd-C (100 mg) and saturated with hydrogen at room temperature and atmospheric pressure. The catalyst was filtered off and the solvent evaporated. The residue obtained after evaporation of the solvent was chromatographed over silica gel column using hexane-EtOAc mixtures as eluent to give pure homoisoflavanones.

(3h, 3i).7-Hydroxy-3-(4-methoxybenzyl)chromanone (3h). Mp: $132-134^{\circ} \mathrm{C}$. Yield 78\% (393mg); Anal.Calcd. for $\mathrm{C}_{17} \mathrm{H}_{16} \mathrm{O}_{4}$ : C, 71.79; H, 5.68\%. Found: C, 71.83; $\mathrm{H}, 5.63 \%$. $\operatorname{IR}(\mathrm{KBr}) v_{\max }: 3216,3005,2836,1657,1602,1334,1207,1158,1124,1040 \mathrm{~cm}^{-1}$; ${ }^{1} \mathrm{H}-\mathrm{NMR}$ $\left(\mathrm{CDCl}_{3}\right): \delta$ 9.72(1H, s, Ar-OH), 7.09(1 H, d, J=8.8 Hz, H-5), 6.84(2H, d, J=8.4Hz, H-2',6'), 6.82 $\left(2 \mathrm{H}, \mathrm{d}, \mathrm{J}=8.4 \mathrm{~Hz}, \mathrm{H}-3^{\prime}, 5^{\prime}\right), \quad 6.34(1 \mathrm{H}, \mathrm{dd}, \mathrm{J}=8.8 \mathrm{~Hz}, 2.4 \mathrm{~Hz}, \mathrm{H}-6), 6.30(1 \mathrm{H}, \mathrm{d}, \mathrm{J}=2.4 \mathrm{~Hz}, \mathrm{H}-8)$, 4.16(1H, dd,J=8.8, 2.8 Hz, H-2a), 4.12(1H, dd, J=8.8, $2.8 \mathrm{~Hz}, \mathrm{H}-2 \mathrm{~b}), 3.79$ (3H,s, Ar-OMe), 2.73 $(1 \mathrm{H}, \mathrm{dd}, \mathrm{J}=4.8,10.8 \mathrm{~Hz}, \mathrm{H}-9 \mathrm{a}), 2.65-2.51(1 \mathrm{H}, \mathrm{m}, \mathrm{H}-3), 2.43(1 \mathrm{H}, \mathrm{dd}, \mathrm{J}=8.8,10.8 \mathrm{~Hz}, \mathrm{H}-9 \mathrm{~b})$; LCMS (ESI, negative ion mode): $m / z 283(\mathrm{M}-\mathrm{H})^{-}$.

7-Hydroxy-3-(2, 4-dimethoxybenzyl)chromanone (3i): Mp: 136-138 ${ }^{\circ} \mathrm{C}$; Yield 75\% (378 mg); Anal.Calcd. for $\mathrm{C}_{18} \mathrm{H}_{18} \mathrm{O}_{5}$ : C, 68.78; H, 5.68\%. Found: C, 68.78; H, 5.63\%.

$\mathrm{IR}(\mathrm{KBr}) v_{\max }: 3214,3001,2836,1652,1582,1334,1207,1158,1124,1040 \mathrm{~cm}^{-1} ;{ }^{1} \mathrm{H}-\mathrm{NMR}$ $\left(\mathrm{CDCl}_{3}\right): \delta 9.85(1 \mathrm{H}, \mathrm{s}, \mathrm{Ar}-\mathrm{OH}), 7.63(1 \mathrm{H}, \mathrm{d}, \mathrm{J}=9.0 \mathrm{~Hz}, \mathrm{H}-5), 6.98(2 \mathrm{H}, \mathrm{d}, \mathrm{J}=7.54 \mathrm{~Hz}, \mathrm{H}-6$ '), $6.31-$ 6.49(3H, m, H-6,3',5'), 6.21(1 H, J=2.1 Hz, H-8), 4.08-4.39(2H, m, H-2a, 2b), 3.81(3H,s, ArOMe), 3.78(3H, Ar-OMe), 3.05-3.19(1H, m, H-9a), 2.80-3.01(1H, m, H-3), 2.61-2.68(1H, m, H9b); LC-MS (ESI, negative ion mode): $m / z 313(\mathrm{M}-\mathrm{H})^{-}$.

\section{Antioxidant activity}

The antioxidant activity of 3-benzylchromone derivatives $(2 \mathbf{a}-\mathbf{i}, \mathbf{3 h} \boldsymbol{\&} \mathbf{3 i})$ was studied by the NBT method ${ }^{25}$. The reaction mixture contained EDTA $(6.6 \mu \mathrm{M}), \mathrm{NaCN}(3 \mu \mathrm{g})$, riboflavin $(2$ $\mu \mathrm{M})$, NBT $(50 \mu \mathrm{M})$, various concentrations of the test drug in ethanol and a phosphate buffer (58 $\mathrm{mM}, \mathrm{pH} 7.8$ ) in a final volume of $3 \mathrm{ml}$. The test tubes were shaken well and the absorbance was 
measured at $560 \mathrm{~nm}$ and mean control OD is 0.8247 . The test tubes were uniformly illuminated with an incandescent lamp for 15 minutes, after which the optical density was measured again at $560 \mathrm{~nm}$. The percent inhibition of superoxide radical generation was measured by comparing the mean absorbance values of the control and those of test substances. IC 50 values were obtained from the plot drawn of concentration in $\mu \mathrm{g}$ versus percentage inhibition and were converted in to $\mu \mathrm{M}$. All the tests were run in triplicate and data were analyzed using one way ANOVA.

\section{Antifungal activity}

The antifungal activity of chromone derivatives (2a-i, $3 \mathbf{h} \boldsymbol{\&} \mathbf{3 i}$ ) was studied by agar cup method $^{26,27}$. Glass Petri dishes used were sterilized and potato dextrose agar was used as basal medium for test fungi. The saboroudes broth medium was prepared by taking peptone $(1.0 \mathrm{~g})$ and dextrose $(4.0 \mathrm{~g})$ in warm distilled water $(100 \mathrm{~mL})$. The selected fungal culture, single colony was inoculated in to broth medium and kept for incubation for overnight at $25{ }^{\circ} \mathrm{C}$. The saboroudes agar medium was prepared by taking peptone $(1.0 \mathrm{~g})$, dextrose $(4.0 \mathrm{~g})$ and agar $(2.0 \mathrm{~g})$ in warm distilled water $(100 \mathrm{~mL})$ and plated into Petri dishes, allowed to solidification. The overnight fungal culture was spread evenly over the entire surface and left undisturbed for few minutes to percolate the culture. Wells $(4 \mathrm{~mm})$ were created using a sterile borer into the solidified agar medium. The selected compounds were added to each well $(10 \& 20 \mu \mathrm{L})$ at peripheral and the reference compound (Fluconazole) was added at the centre. Thus the prepared plates were incubated at room temperature (at about $25{ }^{\circ} \mathrm{C}$ ) for about 3-5 days. After incubation period the plates were collected and record the inhibition zone in $\mathrm{mm}$ (from the margin of the well to surface of inhibition).

Dimethyl sulphoxide (DMSO) was used as solvent to prepare the stock solutions (5 mg in 0.5 $\mathrm{mL}$ ) of the compounds initially and also to maintain proper control. A control well was also placed on the test plates to compare the effect of the test samples and to nullify the effect of solvent (DMSO) respectively.

\section{Acknowledgements}

We are thankful to Prof. D.V.R. Sai Gopal, Dept. of Virology, S.V. University, Tirupati, for providing activity studies at their lab.

\section{References}

1. Boehler, P.; Tamm, Ch. Tetrahedron Lett. 1967, 3479-3483. (b) Finckh, R. E.; Tamm, Ch. Experientia 1970, 26, 472. (c) Heller, W.; Tamm, Ch. Helv. Chem. Acta 1978, 61, 1257. (d) Heller, W.; Andermatt, P.; Schadd, W. A.; Tamm, Ch. Helv. Chem. Acta. 1976, 59, 2048. (e) 
Tada, A; Kasai, R.; Saitoh, T.; Shoji, J. Chem. Pharm. Bull. 1980, 28, 1477. (f) Tada, A.; Kasai, R.; Saitoh, T.; Shoji, J. Chem. Pharm. Bull. 1980, 28, 2039.

2. (a) Saitoh, T.; Sakashita, S.; Nakata, H.; Shimokawa, T.; Kinjo, J. E.; Yamahara, J.; Yamasaki, M.; Nohara, T. Chem. Pharm. Bull. 1986, 34, 2506. (b) McPherson, D. D.; Cordell, G. A.; Soejarto, D. D.; Pezzuto, J. M.; Fong, H. H. S. Phytochemistry 1983, 22, 2835.

3. Ravise, A.; Kirkiacharian, B. S.; Phytopathol. Z. 1978, 92, 36.

4. Al Nakib, T.; Bezak, V.; Meegan, M. J.; Chandy, R, Eur. J. Med. Chem. 1990, $25,455$.

5. Siddaiah, V.; Venkata Rao, C.; Venkateswarlu, S.; Subbaraju, G. V. Tetrahedron 2006, 62, 841.

6. Siddaiah, V.; Venkata Rao, C.; Venkateswarlu, S.; Krishnaraju, A.; Subbaraju, G. V. Bioorganic \& Medicinal Chemistry 2006, 14, 2545.

7. Dermanandan, R.; Dhanutrito, H.; Castel, J.; Loubatierea, J. and Flandre, O. Farmaco 1984, 39, 876.

8. Kirkiacharian, B. S.; Gomis, M.; Koutsourakis, P. G. Eur. J. Med. Chem. 1989, 24, 309.

9. Wall, M. E.; Wani, M. C.; Manikumar, G.; Taylor, H.; Mc Givney, R. J. Nat. Prod., 1989, $52,774$.

10. Anschler, G.; W.Frahm, A.; Hatzelmann, A.; Kilian, U.; Muller-Doblies, D.; Muller-Doblies, U. Planta Medica 1996, 62, 534.

11. Desideri, N.; Oliveri, S.; Stein, M. L;, Sgro, R.; Orsi, N. and Conti, C. Antiviral Chem. Chemother. 1997, 8, 545.

12. Quaglia, M. G.; Desideri, N.; Bossu, E.; Sgro, R.; Conti, C. Chirality 1999, 11, 495.

13. Kirkiacharian, B. S.; Tongo, H. G.; Bastide, J.; Bastide, P.; Grenie, M. M. Eur. J. Med. Chem. 1989, 24, 541.

14. Malhotra, S.; Sharma, V. K.; Parmar, V. S, J. Chem. Res., (S) 1988, 179, 22.

15. Andrieux, J.; Barton, D. H. R.; Patin, H. J. Chem. Soc., Perkin 1 1977, 1359.

16. Chatterjee, J. N.; Shaw, S. C.; Singh, J. N. J. Ind. Chem. Soc. 1974, 51, 28.

17. Farkas, L.; Gottsegen, A.; Nogradi, M. Tetrahedron, 1971, 27, 5049.

18. Bass, R. J. J. Chem. Soc., Chem. Comm. 1976, 78.

19. Jain, A. C.; Mehata, A. Indian J. Chem. 1985, 24B, 1170.

20. Jaspal, S.; Grover, S. K. Indian J. Chem. 2004, 43B, 1782.

21. Kirkiacharian, B. S. J. Chem. Soc., Chem. Commun., 1975, 62.

22. Jaen, J. C.; Wise, L. D.; Heffner, T. G.; Pugsley, T. A.; Meltzer, L. T. J. Med. Chem. 1991, 34, 248.

23. Zhao, P.; Iwamoto, Y; Kouno, I.; Egami, Y.; Yamamoto, H. Phytochemistry 2004, 65, 2455.

24. Jain, A. C.; Anita Sharma, Bikram Singh, Indian J. Chem. 1983, 22B, 759.

25. Mc Cord, J. M.; Fridovich, I. Biol. Chem. 1969, 244, 6049.

26. Pattnaik, S.; Subramanyam, V. R.; Kole, C. Microbios. 1996, 86, 237.

27. Spooner, F. D.; Sykes, G. Laboratory assessment of antibacterial activity. In Methods in Microbiology Norris, E.; Ribbon, D. N., Eds., Academic Press: London, 1972; p 45. 